\title{
Fisioterapia aquática na redução da dor e melhora da qualidade de vida de idosos com osteoartrite de joelho: uma revisão sistemática
}

Aquatic physiotherapy in the reduction of pain and improvement of the quality of life of the elderly with knee osteoarthritis: a systematic review

Fisioterapia acuática para reducir el dolor y mejorar la calidad de vida de las personas mayores con osteoartritis de rodilla: una revisión sistemática

Recebido: 29/10/2021 | Revisado: 07/11/2021 | Aceito: 09/11/2021 | Publicado: 13/11/2021

\author{
Maria Clara Sousa Oliveira \\ ORCID: https://orcid.org/0000-0003-2001-6926 \\ Centro Universitário Santo Agostinho, Brasil \\ E-mail: mariaclarasaky2013@gmail.com \\ Walyssandra Jasmile Campos Veras \\ ORCID: https://orcid.org/0000-0002-9377-2872 \\ Centro Universitário Santo Agostinho, Brasil \\ E-mail: jasmillycampos2@gmail.com \\ Patrícia Lima Ventura \\ ORCID: https://orcid.org/0000-0002-8920-2877 \\ Centro Universitário Santo Agostinho, Brasil \\ E-mail: vlpatricia@hotmail.com
}

\begin{abstract}
Resumo
A osteoartrite é uma disfunção musculoesquelética comumente insidiosa, gradativa e lenta, que se caracteriza pelo desgaste da cartilagem articular e por alterações ósseas. A fisioterapia aquática tem grande relevância no tratamento de pacientes com osteoartrite de joelho, diminuindo a dor e contribuindo para uma melhora significativa na qualidade de vida. O objetivo desse estudo, é revisar sistematicamente a efetividade da fisioterapia aquática na redução da dor em pacientes e melhora da qualidade de vida em idosos com osteoartrite de joelho. Sendo uma revisão sistemática de ensaios clínicos randomizados, analisados por dois revisores independentes, conforme recomendado pela plataforma Prisma nas bases de dados PubMed, PEDro e CENTRAL. Foram incluídos estudos originais utilizando a Fisioterapia Aquática na redução da dor e melhora da qualidade de vida em pacientes idosos com OA de joelho. A escala PEDro foi usada para analisar a qualidade metodológica e análise de risco de viés. Foram incluídos cinco estudos. A Fisioterapia Aquática, interveio na diminuição da dor e melhora da qualidade de vida quando comparado com outros métodos de intervenção aplicados em pacientes idosos com OA de joelho. Conclui-se que a Fisioterapia Aquática é uma opção eficaz para a osteoartrite de joelho, reduzindo os níveis de dor e aumento da qualidade de vida dos idosos.
\end{abstract}

Palavras-chave: Fisioterapia aquática; Osteoartrite de joelho; Qualidade de vida.

\begin{abstract}
Osteoarthritis is a commonly insidious, gradual, and slow musculoskeletal dysfunction characterized by wear of the articular cartilage and bone changes. Aquatic physiotherapy has great relevance in the treatment of patients with knee osteoarthritis, reducing pain and contributing to a significant improvement in quality of life. The aim of this study is to systematically review the effectiveness of aquatic physical therapy in reducing pain in patients and improving the quality of life in elderly patients with knee osteoarthritis. It is a systematic review of randomized clinical trials, analyzed by two independent reviewers, as recommended by the Prisma platform in the PubMed, PEDro and CENTRAL databases. Original studies using Aquatic Physical Therapy to reduce pain and improve quality of life in elderly patients with knee OA were included. The PEDro scale was used to analyze the methodological quality and analysis of risk of bias. Five studies were included. Aquatic Physiotherapy intervened to reduce pain and improve quality of life when compared to other intervention methods applied to elderly patients with knee OA. It is concluded that Aquatic Physical Therapy is an effective option for knee osteoarthritis, reducing pain levels and increasing the quality of life of the elderly.
\end{abstract}

Keywords: Aquatic physiotherapy; Knee osteoarthritis; Quality of life.

\section{Resumen}

La osteoartritis es una disfunción musculoesquelética lenta, gradual y comúnmente insidiosa caracterizada por el desgaste del cartílago articular y cambios óseos. La fisioterapia acuática tiene una gran relevancia en el tratamiento de pacientes con artrosis de rodilla, reduciendo el dolor y contribuyendo a una mejora significativa en la calidad de vida. El objetivo de este estudio es revisar sistemáticamente la eficacia de la fisioterapia acuática para reducir el dolor en los pacientes y 
mejorar la calidad de vida de los pacientes ancianos con osteoartritis de rodilla. Es una revisión sistemática de ensayos clínicos aleatorizados, analizados por dos revisores independientes, según lo recomendado por la plataforma Prisma en las bases de datos PubMed, PEDro y CENTRAL. Se incluyeron estudios originales que utilizan la fisioterapia acuática para reducir el dolor y mejorar la calidad de vida en pacientes ancianos con artrosis de rodilla. Se utilizó la escala PEDro para analizar la calidad metodológica y el análisis del riesgo de sesgo. Se incluyeron cinco estudios. La fisioterapia acuática intervino para reducir el dolor y mejorar la calidad de vida en comparación con otros métodos de intervención aplicados a pacientes ancianos con artrosis de rodilla. Se concluye que la Fisioterapia Acuática es una opción eficaz para la artrosis de rodilla, reduciendo los niveles de dolor y aumentando la calidad de vida de las personas mayores.

Palabras clave: Fisioterapia acuática; Osteoartritis de rodilla; Calidad de vida.

\section{Introdução}

A osteoartrite (OA) é uma disfunção musculoesquelética comumente insidiosa, gradativa e lenta, que acomete principalmente as articulações da coluna, mãos, quadril e joelho. É um distúrbio articular comum, podendo afetar cerca de $6 \%$ a $12 \%$ das pessoas adultas e mais de um terço da população com idade superior a 65 anos. As alterações patológicas da OA são caracterizadas pela perda gradativa da cartilagem articular, podendo afetar também todas as articulações, no qual todos os tecidos são comprometidos: disco intra-articular, ligamento, osso subcondral, cartilagem e estruturas de sustentação (Rosis, Massabki e Kairalla 2010).

A frequência e gravidade da osteoartrite varia em diversas formas, sendo algumas delas a prevalência no sexo feminino e idade avançada. É uma doença crônico-degenerativa frequente no joelho, sendo esta uma articulação de sustentação de peso, provoca uma perda significativa na sua função e consequentemente da qualidade de vida do idoso (Dias, et al. 2017).

Clinicamente, está relacionado a rigidez, dor, deformidade e perda da capacidade funcional. Cerca de $10 \%$ das pessoas com mais de 60 anos é acometida por osteoartrite e 80\% desses indivíduos tem redução de amplitude de movimento. E 25\% têm limitações funcionais que acaba afetando e comprometendo o desempenho das atividades de vida diárias (Alcade, et al. 2017).

A osteoartrite apresenta, geralmente, duas condições: as características dos matérias biológicos da cartilagem articular e do osso subcondral mantém-se normal, e possui uma sobrecarga na articulação, levando a desintegração dos tecidos e; a essência dos materiais da cartilagem e do osso encontram-se alteradas, enquanto há uma carga razoável sobre a articulação (Rosis, Massabki e Kairalla 2010).

A Fisioterapia Aquática proporciona vários benefícios no tratamento da OA de joelho. O calor da água produz um maior relaxamento muscular e articular, aliviando contraturas de tecidos moles, espasmos musculares, fadiga e redução da dor; devido a flutuabilidade ocorre uma diminuição da sobrecarga articular ao entrar na piscina, reduzindo assim o impacto sobre elas. A prática nesse meio torna-se mais fácil para o corpo, dando prazer ao realiza-los melhorando assim sua qualidade de vida (Lu, et al. 2015).

A Fisioterapia é um conjunto de exercícios realizado na água e, vem sendo utilizado desde a antiguidade, proporcionando diversas vantagens devido aos fatores da propriedade da mesma. A temperatura constante em conjunto com a pressão hidrostática ajuda em uma melhor circulação sanguínea (Dong, et al. 2018).

Uma das principais vantagens está atribuída às propriedades físicas e os efeitos fisiológicos possibilitados pelo meio aquático, nos quais frequentemente são recomendadas e receitadas para pacientes com doenças reumáticas como é o caso da osteoartrite de joelho, proporcionando uma gama de serventias incluindo diminuição de edema, melhora do nível da dor e da sobrecarga das articulações já acometidas (Foley, et al. 2003).

As orientações internacionais de prática clínica, apontam a importância de intervenções não farmacológicas, como programas educacionais e de atividades, como recurso terapêutico de primeira linha dentro das opções de tratamento para OA de joelho. Os exercícios podem ser realizados em meio aquático ou terrestre. A fisioterapia aquática é bastante recomendada como opção de tratamento para pessoas com idade avançada, realizado em um ambiente seguro e com menor risco de quedas. Esses pacientes apresentam níveis mais elevados de adesão ao tratamento com o recurso utilizado (Dias, et al. 2017). 
O prezado estudo tem por objetivo a realização de uma revisão sistemática da literatura, analisando a efetividade da fisioterapia aquática na redução da dor em pacientes idosos com osteoartrite de joelho e melhora da qualidade de vida dos mesmos.

\section{Metodologia}

Consiste em um estudo de revisão sistemática, de caráter qualitativo. Essa revisão buscar responder a seguinte pergunta clínica: “A fisioterapia aquática é efetiva na redução da dor e melhora da qualidade de vida de idosos com osteoartrite de joelho?" Para responder essa pergunta, partiu-se da metodologia/estratégia PICO (Population/População; Intervention/Intervenção; Comparison/Comparação; Outcomes/Desfecho).

P: Pacientes idosos com AO de joelho;

I: Fisioterapia Aquática;

C: Nenhuma Intervenção;

O: Redução da dor e melhora da qualidade de vida.

Foi realizado uma busca dos artigos através de pesquisas nas bases de dados PEDro, PubMed e CENTRAL, não havendo restrição quanto ao ano e idioma, utilizou os seguintes descritores e operadores booleanos "Aquatic Physiotherapy" AND "Knee osteoarthritis" AND "Elderly" AND "Quality of life".

Foram incluídos apenas ensaios clínicos randomizados que abordavam a efetividade da fisioterapia aquática em pacientes idosos com diagnóstico clínico de osteoartrite da articulação do joelho e excluímos estudos que abordavam outras patologias ou artigos que não traziam a fisioterapia aquática como forma de intervenção.

A qualidade metodológica desses artigos utilizou a escala PEDro, desenvolvida pela Physiotherapy Evidence Database, empregada em ensaios clínicos e tem uma pontuação total de até 10 pontos, incluindo critérios de avaliação de validade interna e apresentação da análise estatística empregada. Busca quantificar a qualidade dos estudos publicados, de forma a guiar os usuários sobre os aspectos meritórios de cada publicação e facilitar a identificação rápida de estudos que contenham informações suficientes para a prática profissional. Essa escala é composta por 11 critérios e a pontuação final é gerada através da somatória de dez dos onze critérios.

A seleção dos estudos para esta revisão sistemática, foi realizada por dois revisores independentes, a leitura do título e resumo foi o ponto de partida utilizado para a seleção dos artigos, seguida pela avaliação dos critérios de inclusão e exclusão em cada estudo, com intuito de obter resultados mais criteriosos para a construção dessa revisão sistemática. Os estudos foram separados e catalogados para que pudessem ser comparados e discutidos entre os pesquisadores, e ao final, ficaram apenas os estudos que atenderam aos critérios de elegibilidade da escala PEDro, para que o objetivo deste estudo fosse alçando.

\section{Resultados}

O pontapé inicial para o início dessa metodologia partiu-se da metodologia/estratégia PICO. Deixando claro qual a patologia a ser tratada, qual o tipo de intervenção e quais seria os resultados encontrados durante a pesquisa. 
Quadro 1 - Estratégia de pesquisa PICO. Teresina-PI, 2021.

\begin{tabular}{|c|c|c|}
\hline ACRÔNIMO & DEFINIÇÃO & DESCRIÇÃO \\
\hline P & População & Pacientes idosos com osteoartrite de \\
& & joelho \\
\hline I & Intervenção & Nenhum \\
\hline C & Controle & Redução da dor crônica e melhora na \\
qualidade de vida
\end{tabular}

Fonte: Autores.

Registros buscados nas bases de dados: PEDro, CENTRAL e PUBMED, durante a pesquisa foram encontrados no total de 154 artigos, utilizando os descritores fisioterapia aquática AND osteoartrite de joelho AND idosos AND qualidade de vida. Foi utilizado ainda os filtros: ensaios clínicos randomizados e textos completos, não houve restrição de idioma e/ou período de publicações como critérios de inclusão e exclusão, reduzindo assim para 125 artigos.

Após a leitura do título e resumo 105 registros foram excluídos e apenas 20 foram selecionados. Após uma leitura minuciosa dos resultados apenas 14 artigos foram selecionas e 9 artigos foram excluídos pois não respondiam ao objetivo da pesquisa, chegando ao final apenas 5 artigos incluídos na síntese qualitativa. 
Fluxograma 1. Pesquisa e triagem dos estudos relacionados nos bancos de dados, conforme a plataforma PRISMA. TeresinaPI, 2021.

\begin{tabular}{|c|}
\hline 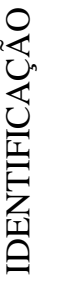 \\
\hline
\end{tabular}
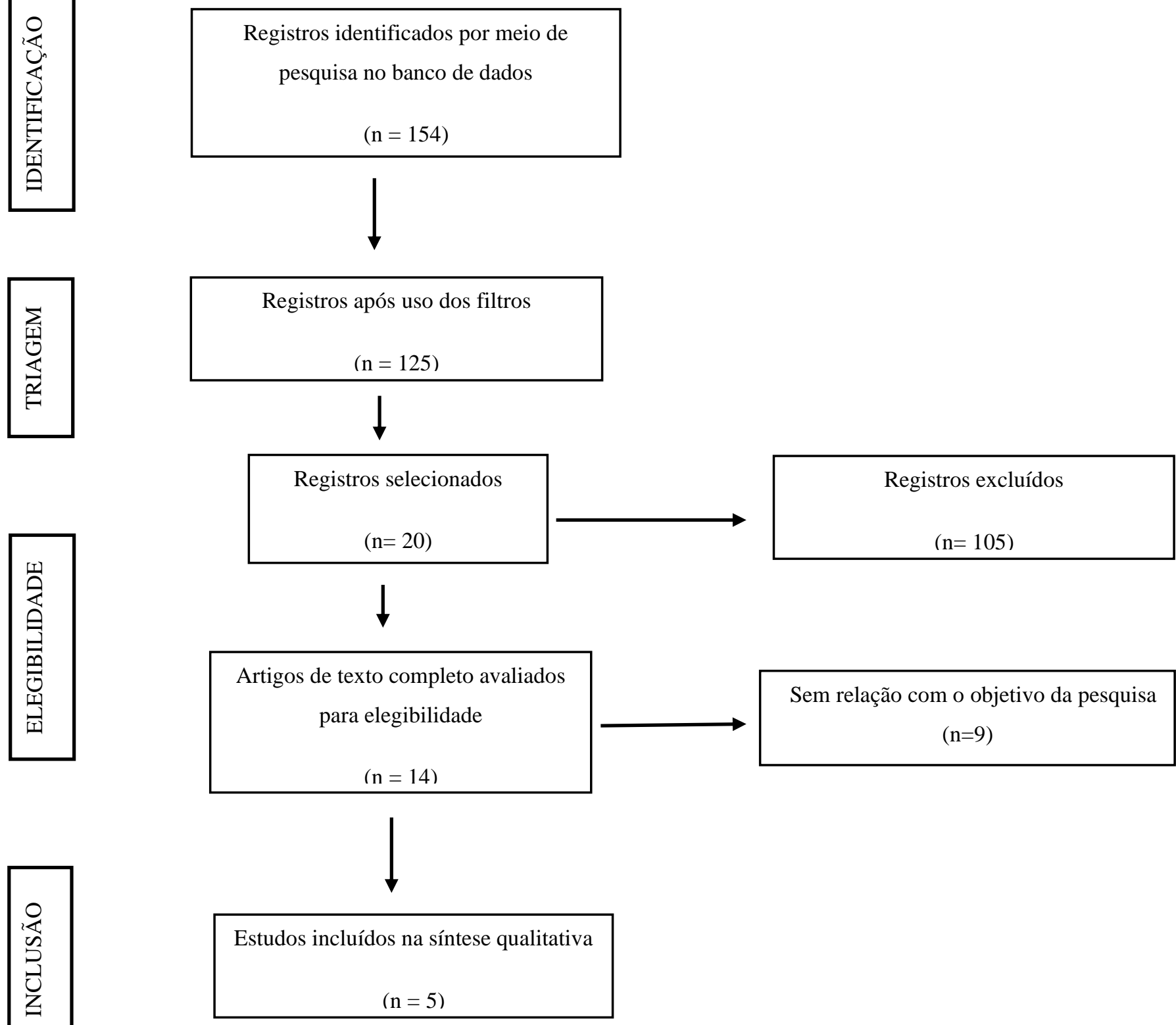

Fonte: Autores.

Após a seleção dos artigos, foi realizada uma análise da qualidade metodológica desses artigos usando a ferramenta de avaliação crítica PHYSIOTHERAPY EVIDENCE DATABASE (PEDro), com o intuito de analisar o risco de viés dessa revisão sistemática, baseado na pontuação de score em cada item analisado do artigo. 
Quadro 2. Análise da qualidade metodológica dos estudos por meio da escala PEDro. Teresina-PI, 2021.

\begin{tabular}{|c|c|c|c|c|c|}
\hline & $\begin{array}{l}\text { Azizi et al. } \\
2019\end{array}$ & $\begin{array}{l}\text { Hinman et al. } \\
2007\end{array}$ & $\begin{array}{l}\text { Taglietti et al. } \\
2018\end{array}$ & $\begin{array}{l}\text { Silva et al. } \\
\quad 2008\end{array}$ & $\begin{array}{l}\text { Khruakhorn et al. } \\
\qquad 2021\end{array}$ \\
\hline 1 Critério de elegibilidade e origem & SIM & SIM & SIM & SIM & NÃO \\
\hline 2 Distribuição aleatória & SIM & SIM & SIM & SIM & SIM \\
\hline 3 Distribuição escondida & SIM & SIM & SIM & NÃO & SIM \\
\hline 4 Comparação ao ponto de partida & SIM & SIM & SIM & SIM & NÃO \\
\hline 5 Cegamento de sujeitos & NÃO & NÃO & NÃO & NÃO & NÃO \\
\hline 6 Cegamentos de terapeutas & NÃO & NÃO & NÃO & $\mathrm{NA} O$ & NÃO \\
\hline 7 Cegamento de avaliadores & SIM & SIM & NÃO & SIM & SIM \\
\hline 8 Avaliação> $85 \%$ & SIM & SIM & SIM & SIM & SIM \\
\hline $\begin{array}{l}9 \text { Análise por internação de } \\
\text { tratamento }\end{array}$ & SIM & SIM & SIM & SIM & SIM \\
\hline 10 Comparação inter-grupos & SIM & SIM & SIM & SIM & NÃO \\
\hline $\begin{array}{l}11 \text { Medidas de precisão e } \\
\text { variabilidade }\end{array}$ & SIM & SIM & SIM & SIM & SIM \\
\hline SCORE & $8 / 10$ & $8 / 10$ & $7 / 10$ & $7 / 10$ & $6 / 10$ \\
\hline
\end{tabular}

Fonte: Autores.

Após a análise dos artigos, os estudos selecionados foram organizados em um quadro para apresentação do perfil das publicações, afim de tornar mais fácil o entendimento dos mesmos. O quadro a seguir apresenta os estudos caracterizados de acordo com a pontuação final no nível de score. Os artigos encontrados foram publicados em 2007, 2008, 2018, 2019 e o mais recente, em 2021. Azizi et al. 2019, Hinman et al. 2007, Taglietti et al. 2018, e Silva et al. 2008, foram encontrados na base de dados PEDro. Khruakhorn et al. 2021, foi o único encontrado na base de dados PubMed, avaliados com a escala PEDro, e todos os artigos estavam no idioma inglês. 
Quadro 3. Dados gerais sobre os artigos selecionados para obtenção dos resultados. Teresina-PI, 2021.

\begin{tabular}{|c|c|c|c|c|}
\hline Autor & Amostra & Objetivos & Intervenção & Resultados \\
\hline Azizi, et al. 2019 & 32 & $\begin{array}{l}\text { O objetivo deste estudo é } \\
\text { avaliar a eficácia do } \\
\text { exercício aquático na dor, } \\
\text { marcha e equilíbrio em } \\
\text { pacientes idosos com } \\
\text { osteoartrite de joelho }\end{array}$ & $\begin{array}{c}\text { Intervenção - realizou } 3 \\
\text { sessões de exercícios } \\
\text { aquáticos durante } 8 \text { semanas } \\
\text { Controle - usou paracetamol e } \\
\text { seguiu o estilo de vida } \\
\text { recomendado }\end{array}$ & $\begin{array}{l}\text { O grupo de intervenção, mostrou alívio } \\
\text { da dor }(p=0,019) \text {, além de melhora } \\
\text { significativa ao equilíbrio estático e } \\
\text { dinâmico }(p=0,001) \text {, comprimento do } \\
\text { passo }(p=0,038) \text {, comprimento da } \\
\text { passada e cadência }(p<0,001)\end{array}$ \\
\hline $\begin{array}{c}\text { Hinman et al. } \\
2007\end{array}$ & 71 & $\begin{array}{l}\text { O objetivo deste estudo foi } \\
\text { avaliar os efeitos da } \\
\text { Fisioterapia Aquática na } \\
\text { OA de quadril e/ou joelho }\end{array}$ & $\begin{array}{l}\text { Intervenção - realizaram } \\
\text { Fisioterapia Aquática durante } \\
6 \text { semanas } \\
\text { Controle - não recebeu } \\
\text { nenhum tipo de intervenção }\end{array}$ & $\begin{array}{l}\text { Totais de } 72 \% \text { e } 75 \% \text { dos participantes } \\
\text { do grupo intervenção, relataram } \\
\text { melhorias na dor, função, força } \\
\text { muscular e qualidade de vida, } \\
\text { comparado ao grupo controle }\end{array}$ \\
\hline $\begin{array}{c}\text { Taglietti et al. } \\
2018\end{array}$ & 60 & $\begin{array}{l}\text { Comparar a eficácia dos } \\
\text { exercícios aquáticos com a } \\
\text { educação do paciente em } \\
\text { indivíduos com } \\
\text { osteoartrite de joelho }\end{array}$ & $\begin{array}{l}\text { Intervenção - protocolo de } \\
\text { tratamento de } 8 \text { semanas de } \\
\text { exercícios aquáticos ( } 16 \\
\text { sessões individuais, } 2 \text { vezes } \\
\text { por semana) } \\
\text { Controle - programa } \\
\text { educacional (sessões em } \\
\text { grupo, uma vez por semana) }\end{array}$ & $\begin{array}{l}\text { No final do tratamento, foi notado uma } \\
\text { melhora significativa em relação a dor, } \\
\text { função e qualidade de vida } \\
\text { em comparação com o grupo do } \\
\text { Programa Educacional, } \\
\text { MD = }-3,8 \text { pontos; IC de } 95 \%(-8,7 ;- \\
1),(P=0,021) .\end{array}$ \\
\hline Silva, et al. 2008 & 64 & $\begin{array}{l}\text { Avaliar a eficácia da } \\
\text { Fisioterapia Aquática em } \\
\text { indivíduos com OA de } \\
\text { joelho em comparação a } \\
\text { indivíduos com OA de } \\
\text { joelho que realizavam } \\
\text { exercícios em solo }\end{array}$ & $\begin{array}{l}\text { Intervenção - realizou por } 18 \\
\text { semanas exercícios aquáticos } \\
\text { Controle - realizou exercícios } \\
\text { em solo por } 18 \text { semanas }\end{array}$ & $\begin{array}{l}\text { Ambos os grupos melhoraram a dor e a } \\
\text { funcionalidade do joelho. Contudo, } \\
\text { apenas a Fisioterapia Aquática } \\
\text { demonstrou melhora desde o início até } \\
\text { o final do estudo. }\end{array}$ \\
\hline $\begin{array}{l}\text { Khruakhorn et al. } \\
2021\end{array}$ & 34 & $\begin{array}{c}\text { Determinar os efeitos da } \\
\text { hidroterapia e exercícios } \\
\text { terrestres na mobilidade } \\
\text { funcional e na qualidade } \\
\text { de vida de pacientes com } \\
\text { osteoartrite de joelho }\end{array}$ & $\begin{array}{c}\text { Intervenção - praticaram por } 6 \\
\text { meses de fisioterapia aquática } \\
\text { Controle - praticaram } \\
\text { exercícios terrestres por } 6 \\
\text { meses }\end{array}$ & $\begin{array}{l}\text { O grupo intervenção (Fisioterapia } \\
\text { Aquática) mostrou melhora } \\
\text { significativa nos domínios mental, } \\
\text { social e qualidade de vida, após } 6 \\
\text { meses. }\end{array}$ \\
\hline
\end{tabular}

Fonte: Autores.

\section{Discussão}

Para esta revisão sistemática, foram encontrados cinco artigos nas bases de dados (PEDro, PUBMED e CENTRAL), que avaliaram os benefícios da Fisioterapia Aquática na redução da dor e melhora da qualidade de vida de pacientes idosos com OA de joelho. Assim, tendo o objetivo de investigar sua eficácia como intervenção, comparando-o com outros métodos, como: 
exercícios em solo, programa educacional, tratamento medicamentoso e mudanças de hábito de vida, além de nenhum tipo de intervenção.

Todos os artigos estudados utilizaram a Fisioterapia Aquática como intervenção. Azizi, et al., 2019, o comparou com o uso do paracetamol para alívio das dores e mudanças do hábito de vida para a qualidade de vida; Hinman et al., 2007 foi o único que não utilizou nenhum tipo de comparação no Grupo Controle (GC); Taglietti et al., 2018 utilizou as escalas Escala Visual Analógica (EVA) e WOMAC para avaliação entre os grupos e no (GC) participaram de um programa educacional; Silva, et al. também utilizou escalas, elas sendo WOMAC e Lasquene; Silva, et al., 2008 e Khruakhorn et al., 2021 fez comparação com a prática de exercícios terrestres no grupo controle.

Azizi et al., 2019, usou como intervenção a Fisioterapia Aquática, três sessões por 8 semanas, em comparação ao grupo controle que foi receitado paracetamol e mudanças no hábito de vida, no início do estudo não foi encontrada diferenças nos níveis de dor entre os dois grupos, baseado na escala EVA, porém no final do estudo a diferença foi significativa em favor do grupo intervenção, reduzindo os níveis de dor, melhora da marcha e equilíbrio, não mostrando ainda qualquer efeito adverso quanto à prática do exercício em meio aquático.

Hinman et al., 2007, em seu método, reconheceu que um programa de 2 sessões por 6 semanas, leva à redução da dor e rigidez das articulações, bem como a melhora física, força muscular e qualidade de vida de pessoas com osteoartrite de joelho. Além disso, os benefícios da fisioterapia aquática foram mantidos 6 semanas após a conclusão do programa supervisionado. Foi recomendado ainda que tanto o grupo intervenção, quanto o grupo controle, que não recebeu nenhum tipo de programa de tratamento, realizassem a Fisioterapia Aquática, mesmo após o estudo.

Taglietti et al., 2018, comparam dois métodos diferentes, um protocolo de 8 semanas de exercícios aquáticos e um programa educacional. Ao final do acompanhamento, o estudo mostrou que a prática da Fisioterapia Aquática quando comparado com o programa educacional do paciente, mostra-se superior na redução da dor, melhora da função e qualidade de vida em indivíduos com OA de joelho.

Um programa de 18 semanas foi realizado por Silva et al., 2008, entre exercícios aquáticos e exercícios terrestres. Ambos os grupos demostraram redução a dor e melhora da função do joelho conforme medido pelo WOMAC e o Lequesne. Curiosamente, apenas o grupo de fisioterapia aquática continuou a mostrar melhorias nestes índices até o final do estudo.

Khruakhorn et al., 2021, comparou os benefícios da Fisioterapia Aquática com o exercício em solo por 6 meses. Apesar de serem feitos em ambientes diferentes, foi usada a base dos mesmos exercícios entres os grupos. Após a avaliação de 6 semanas e 6 meses não foi notada diferença entre o grupo intervenção e GC. Porém usando a escala WOMAC, foi estabelecida a melhora significativa em favor da Fisioterapia Aquática na qualidade de vida dos pacientes.

No geral, Azizi et al., 2019; Hinman et al., 2007; Taglietti et al., 2018; Silva, et al., 2008 e Khruakhorn et al., 2021 concordam que a Fisioterapia Aquática, quando comparada com outras técnicas aplicadas, é uma opção eficaz no tratamento da osteoartrite de joelho em idosos. No entanto, todos os estudos incluídos nesta revisão sistemática, foram parâmetros encontrados após a intervenção nos pacientes.

\section{Conclusão}

Esse estudo mostra que a Fisioterapia Aquática como um programa de intervenção para a osteoartrite de joelho em idosos, apresenta resultados positivos na redução da dor e melhora da qualidade de vida dos pacientes, além da melhora da capacidade funcional, equilíbrio, marcha e aumento da força muscular. Sendo uma opção eficaz para o tratamento da OA de joelho. 


\section{Referências}

Alcade, G. E., et al. (2017). Effect of aquatic physical therapy on pain perception, funcional capacity and quality of life in older people with knee osteoarthritis: study protocol for a randomized controlled trial. BioMed Central, 1-6.

Azizi, S. A. D., Zahra, R., S. A. Raeissadat, R. K. M., \& Sharif, N. (2019). Randomized controlled trial of aquatic exercise for treatment of knee osteoarthritis in elderly people. Interventional Medicine \& Applied Science, 161-167.

Campion M. R. (1997) Hydrotherapy: principles and practice, 2nd edn. Butterworth-Heinemann, Oxford.

Souza, D.V., Lasmar, S. M., Abreu, R. K., Ramires, J. B., Arêas, Gpt. \& Borges, G. F. (2013). Exercise and osteoarthrosis: a systematic review [in Portuguese]. Fisioter Mov. 26:193-202.

Dias, J. M., et al. (2017). Hydrotherapy improves pain and function in older women with knee osteoarthritis: a randomized controlled trial. Brazilian Journal of Physical Therapy, 1-8.

Dong, R. et al. (2018). Is aqautic exercise more effective than land-based exercise for knee osteoarthritis? Medicine, 1-13.

Foley, A., J. Halbert, T. Hewitt, \& M, Crotty. (2003). Does hydrotherapy improvise strength and physical function in patients with osteoarthritis - a randomized controlled trial comparing a gym based and a hydrotherapy based strengthening programme. Ann Rheum Dis, 1162-1167.

Hinman, R. S, S. E. Heywood, \& Anthony, R. D. (2007). Aquatic Physical Therapy for Hip and Knee Osteoarthritis: Results of a Single-Blind Randomized Trial. Research Report, 32-43.

Hochberg, M., Altman, R. \& April, K., et al. (2012). American college of rheumatology recommendations for the use of nonpharmacologic and pharmacologic therapies in osteoarthritis of the hand, hip, and knee. Arthritis Care Res (Hoboken). 64:465---474.

Kelley, W. N. \& Harris, E. D, (2005). Kelley's Textbook of Rheumatology. 7th ed, Philadelphia: Elsevier Saunders.

Khruakhorn, S. \& Sanon, C. (2021). Effects of hydrotherapy and land-based exercise on mobility and quality of life in patients whith knee osteoarthritis: a randomized control trial. The Journal of Physical Therapy Sciense, 375-383.

Lim, J. Y., Tchai, E. \& Jang, S. N. (2010). Effectiveness of aquatic exercise for obese patients with knee osteoarthritis: a randomized controlled trial.” The jornal of injury, function and rehabilitation, 723-731.

Lu, M., et al. (2015). Effectiveness of aquatic exercise for treatment of knee osteoarthritis. Zeitschrift für Rheumatologie, 543-552.

Rosis, R. G. P. Sérgio, M., \& Maisa, K. (2010). Osteoartrite: avaliação clínica e epidemiológica de pacientes idosos em instituição de longa permanência.” Rev Bras Clin Med, 101-108.

Silva, L. E., et al. (2008). Hydrotherapy Versus Conventional Land-Based Exercise of the Management of Patients With Osteroarthritis of the Knee: A Randomized Clinical Trial." Physical Terapy, 12-21.

Simon, L. \& Blotman, F. (1981). Exercise therapy and hydrotherapy in the treatment of the rheumatic diseases. Clin Rheum Dis 7:337-47.

Taglieti, M., et al. (2018). Efevtiveness of aquatic exercises compared to patient-education on health status in individuals with knee osteoarthritis: a randomized controlled trial." Clinical Rehabilitation, 1-11.

Vanni, G. F, Stucky, J. M. \& Schwarstmann, C. A. (2008). Radiologic evaluation of joint space in hip arthrosis: comparative study in decubitus and upright [in Portuguese]. Rev Bras Ortop. 43:460-4.

Vannucci, A. (2002). Osteoartrose. Ver Bras Med. 20(1):36-43.

Wang, X. G, et al. (2013). Effects of tai chi program on neuromuscular function for patients with knee osteoarthritis: study protocol for a randomized controlled trial. Trials. 14:375.

Zhang, W., Doherty, M. \& Arden, N., et al. (2005). EULAR evidence based recommendations for the management of hip osteoarthritis: report of a task force of the EULAR Standing Committee for International Clinical Studies Including Therapeutics. Ann Rheum Dis. 64:669---681. 